\title{
Use of a single-code/default strategy by pigeons to acquire duration sample discriminations
}

\author{
REBECCA A. SINGER, EMILY D. KLEIN, and THOMAS R. ZENTALL \\ University of Kentucky, Lexington, Kentucky
}

\begin{abstract}
Past evidence that pigeons may adopt a single-code/default strategy to solve duration sample discriminations may be attributable to the similarity between the intertrial interval (ITI) and the retention interval. The present experiments tested whether pigeons would adopt a single-code/default strategy when possible ITI-retention-interval ambiguity was eliminated and sample salience was increased. Previous studies of duration sample discriminations that have purported to show evidence for the use of a single-code/default coding strategy have used durations of 0,2 , and $10 \mathrm{sec}$ (Zentall, Klein, \& Singer, 2004). However, the results of Experiment 1 suggest that the use of a 0-sec sample may produce an artifact resulting in inadvertent present/absent sample matching. In Experiment 2, when pigeons were trained with three nonzero duration samples (2, 8, and $32 \mathrm{sec})$, clear evidence for the use of a single-code/default strategy was found.
\end{abstract}

Duration sample discriminations typically involve a conditional discrimination in which one comparison stimulus is correct after a short-duration sample (e.g., $2 \mathrm{sec})$ and the alternative comparison is correct after a long-duration sample (e.g., $10 \mathrm{sec})$. Following acquisition, memory for these durations may be tested by inserting a delay between the offset of the sample and the onset of the comparisons. Typically, with increasing delays, pigeons' memory for the short (2-sec) sample remains consistently high, but memory for the long (10-sec) sample declines, often below chance (Spetch, 1987). This divergence of retention functions has been called the choose-short effect, because after long delays, pigeons almost exclusively choose the comparison associated with the short-duration sample.

One proposed explanation of the choose-short effect is the subjective-shortening hypothesis (Spetch \& Wilkie, 1983; see also Spetch, 1987). According to this hypothesis, over time, memory for the sample duration progressively shortens. Thus, after a long retention interval, memory for a long-duration sample more closely resembles the memory for the short-duration sample experienced during training (when there was no delay). Memory for the short-duration sample also shortens with increasing retention interval; however, it is still more similar to the short-duration sample than to the long-duration sample, so matching accuracy does not decrease on these trials.

It should be noted, however, that duration sample discriminations do not always yield divergent retention functions. For example, if pigeons are trained on a more complex conditional discrimination to choose one comparison

This research was supported by National Institute of Mental Health Grant MH-63726. E.D.K. is now at the Language Research Center, Georgia State University, Atlanta. Correspondence concerning this article should be sent to R. A. Singer, Department of Psychology, University of Kentucky, Lexington, KY 40506-0044 (e-mail: rasing2@uky.edu). stimulus when the sample is either of short duration or vertical lines and to choose the other comparison stimulus when the sample is either of long duration or horizontal lines, when retention intervals are inserted between the sample and the comparison stimuli, the resulting retention functions are parallel not only on line orientation sample trials, but also on duration sample trials (Grant \& Spetch, 1993). This result suggests some flexibility on the part of pigeons and suggests that the way in which duration samples are represented may depend on the context in which the task is acquired.

Divergent retention functions have also been found when the samples were not durations but, rather, the presence versus the absence of a stimulus. For example, Grant (1991) trained pigeons on a conditional discrimination, with food and the absence of food serving as samples. One comparison was correct following the presentation of food, and the alternative comparison was correct when food had not been presented. During testing with retention intervals, matching accuracy on no-food-sample trials remained highly accurate with increasing retention interval, and matching accuracy on food-sample trials declined rapidly with increasing retention interval (see also Sherburne \& Zentall, 1993). Thus, retention functions for food and no-food samples look quite similar to those obtained in typical duration discrimination experiments.

The similarity in the divergent retention functions found following duration sample discriminations and those following present/absent sample discriminations suggest that they may result from similar underlying mechanisms. A number of investigators have suggested that the divergent retention functions found following present/absent sample discrimination training can be attributed to an asymmetrical coding process in which the animals code or represent only one of the samples (the one that is presented) and that they choose the alternative comparison by default, in the absence of a code for the present sample (Colwill, 1984; Grant, 1991; 
Wilson \& Boakes, 1985). This hypothesis, known as the single-code/default hypothesis, is very similar in mechanism to Gaitan and Wixted's (2000) detection strategy.

Gaitan and Wixted (2000) proposed that during training, pigeons may be detecting, or coding, only the most salient sample. In the case of present/absent sample matching, the most salient sample would be the present sample. Therefore, on present-sample trials, if the pigeon remembered the present sample, it would choose the correct comparison, but if it could not remember the present sample, it would, by default, systematically choose the comparison associated with the absent sample. On absentsample trials, the pigeon would continue to choose the comparison associated with the absent sample by default at all retention intervals, because it would have no memory for the present sample.

According to Gaitan and Wixted (2000), in the case of duration sample matching, the most salient sample would be the long (10-sec) duration sample. On test trials, if the pigeon remembered that a 10 -sec sample had been presented, it would choose the comparison associated with the 10-sec sample. However, if it could not remember the 10 -sec sample, it would choose the alternative comparison by default. Once again, on the short (2-sec) sample trials, comparison choice would occur by default in the absence of a memory for the (10-sec) sample.

Gaitan and Wixted (2000) proposed that pigeons may adopt a detection strategy, such as single-code/default, whenever such a strategy provides them with an efficient means of acquiring the task. Clement and Zentall (2000) tested this hypothesis with matching to sample, using hue samples. When one sample (e.g., a yellow hue) was associated with choice of one comparison and each of several other hue samples (e.g., red, blue, green, and white) was associated with choice of the other comparison, the pigeons appeared to code and remember only the yellow sample. On trials involving one of the nonyellow hue samples, the pigeons appeared to choose the alternative comparison by default. Evidence for the use of a single-code/default coding strategy came in the form of divergent retention functions with increasing retention interval. Specifically, Clement and Zentall found declining matching accuracy on yellow sample trials, but not on other sample trials. More directly, when Clement and Zentall presented the pigeons with a novel sample, the pigeons showed a strong tendency to choose the default comparison.

In a similar vein, Gaitan and Wixted (2000) proposed that if two duration samples were associated with one comparison (e.g., red) and a third duration sample was associated with the alternative comparison (e.g., green), a detection strategy might result in which the single duration associated with the green comparison would be coded. On trials on which one of the other two sample durations was presented, the pigeon would choose the red comparison by default. Gaitan and Wixted (2000, Experiment 2) found specifically that when 0 - and 2-sec samples were associated with one comparison and 10-sec samples were associated with the other comparison, divergent retention functions were found, with accuracy on 0 - and 2-sec sample trials remaining consistently high but accuracy on 10-sec sample trials dropping below chance with increasing retention interval. Although these results are consistent with the use of a single-code/default strategy, given the similarity between the 0 - and the 2 -sec samples, these results are also compatible with the subjective-shortening account.

However, in a follow-up experiment, Gaitan and Wixted (2000, Experiment 3) found similar divergent retention functions when 0 - and 10 -sec samples were associated with one comparison and 2-sec samples were associated with the other. They found excellent matching accuracy on 0 - and 10 -sec sample trials and declining matching accuracy on 2-sec sample trials. This result is consistent with a single-code/default strategy but is inconsistent with the subjective-shortening hypothesis, which predicts that at intermediate retention intervals, the subjective duration of the 10-sec sample should appear more similar to the reference memory (from training) for the 2-sec sample, resulting in the erroneous choice of the comparison associated with the 2-sec sample at longer retention intervals.

It is important to note that Gaitan and Wixted (2000) used both dark intertrial intervals (ITIs) and dark retention intervals. However, Sherburne, Zentall, and Kaiser (1998) have suggested that many of the effects found with duration sample matching, such as those found by Gaitan and Wixted, may be attributable to the ambiguity caused by the similarity between the ITI used in training and the novel retention interval used in testing. Specifically, if the ITI and the retention interval are similar in appearance, then on test trials, pigeons may treat the novel retention interval as an ITI, and the subsequent appearance of the comparisons may be viewed as a trial with no sample. Similarly, the choose-short effect observed in duration sample tasks with 2- and 10-sec samples may result from the retention interval's being treated as an ITI and, thus, as a trial with no sample. Because no-sample is more similar to a $2-\mathrm{sec}$ sample than to a 10 -sec sample, after a retention interval, pigeons would choose the comparison associated with the 2 -sec sample. This confusion between the ITI and the retention interval may also explain results obtained in Gaitan and Wixted's three-duration-sample discriminations. In training, Gaitan and Wixted's pigeons learned to select one comparison after a 0 -sec sample (e.g., darkness); therefore, during testing, the dark retention intervals were similar to 0 -sec samples, resulting in choice of the comparison associated with the 0 -sec sample (incidentally associated with the same comparison as the 2-sec sample in their Experiment 2 and the 10-sec sample in their Experiment 3).

Sherburne et al. (1998) suggested that ambiguity between the ITI and the retention interval might be resolved by clearly distinguishing between the two intervals. They tested this hypothesis by manipulating the similarity of the ITI and the retention interval. One group of pigeons had dark ITIs and dark retention intervals (Group Dark), whereas a second group of pigeons had lit ITIs and dark retention intervals (Group Lit). All of the pigeons were trained to choose one comparison after a 2-sec sample and the alternative comparison after a 10 -sec sample. The pigeons were then tested with various retention intervals. 
Divergent retention functions were found only when both the ITI and the retention interval were dark. When the ITIs were lit and the retention intervals dark, the retention functions were quite parallel (but see Kelly \& Spetch, 2000). Similar results were obtained by Zentall, Klein, and Singer (2004) using three sample durations.

Clearly, pigeons do not always code duration samples asymmetrically. But this does not mean that under all unambiguous conditions, parallel retention functions will be found. Recently, Wixted and Gaitan (2004) have suggested that the salience of duration samples may play a role in their detection and, hence, in the likelihood of finding divergent retention functions suggestive of the use of a detection or single-code/default strategy. In this regard, it is interesting that when parallel retention functions have been found following training with duration sample matching, the samples used have been relatively nonsalient. In the case of Sherburne et al.'s (1998) experiments, the durations were signaled by a white response key. Since the immediately preceding event was a relatively bright white houselight, the duration samples would be considered relatively nonsalient. Similarly, in the case of Zentall et al.'s (2004) experiments, although the durations were signaled by a diffuse blue houselight, it was relatively dim when compared with the immediately preceding bright white houselight.

Perhaps if the sample durations were signaled by a more salient event than the event that signaled the ITI, the pigeons would be more likely to use a single-code/default (or detection) strategy, and asymmetrical retention functions would result, even if the ITI and retention intervals were discriminably different. To accomplish this, in Experiment 1, we reversed the function of the two houselights used by Zentall et al. (2004). That is, we used the dimmer, blue houselight during the ITI and the brighter, white houselight as the duration samples. Thus, the purpose of Experiment 1 was to determine whether, when there was no ambiguity between the ITI and the retention interval and the duration samples were more salient than those used by Zentall et al., asymmetrical retention functions would be found (rather than the parallel retention functions found by Zentall et al.).

\section{EXPERIMENT 1}

Given three duration samples (0, 2, and $10 \mathrm{sec})$, the ambiguity hypothesis proposed by Zentall et al. (2004) predicts that when the ITI and the retention intervals are similar in appearance and pigeons code all three samples, they will respond during test as though retention intervals, especially longer ones, were 0-sec samples. Such behavior would result in divergent retention functions. However, it is also possible that rather than coding all three samples, the pigeons could use a more efficient single-code/default strategy, which would also produce divergent retention functions. One way to distinguish between divergent retention functions resulting from the ambiguity of the retention interval and those resulting from a single-code/default strategy is to pair 2- and 10-sec samples with one comparison and 0 -sec samples with the other comparison. Under ambigu- ous conditions, responding to the 0 -sec comparison should remain high, whereas responding to the 2- and 10-sec associated comparison should decline (because the pigeons would be treating the retention intervals as 0 -sec samples). However, under disambiguated conditions, the use of a single-code/default strategy would predict quite different retention functions. It would be more efficient to code the 0 -sec samples and, on 2- and 10-sec sample trials, choose the alternative comparison by default. Specifically, with an increasing retention interval, accuracy on 0 -sec (or singlesample) trials should decrease, whereas accuracy on 2- and 10 -sec sample trials should remain high. This outcome is expected because, in the absence of memory for a 0 -sec trial, the comparison associated with 2 and $10 \mathrm{sec}$ would be selected by default. Representations of these hypothesized outcomes can be seen in Figure 1.

Method
Subjects
The subjects were 8 White Carneaux pigeons (Columba livia)
that were retired breeders (5-8 years of age) purchased from the
Palmetto Pigeon Plant (Sumter, SC). The pigeons were individually
housed in wire cages and maintained at $85 \%$ of their free-feeding
body weights for the duration of the experiment. The pigeons had
free access to water and grit in their home cages. The pigeon colony
room was maintained on a 12:12-h light:dark cycle. The pigeons
were cared for in accordance with University of Kentucky animal
care guidelines. All the pigeons had previous experience in unrelated
studies involving simple simultaneous discriminations and two-
sample, two-alternative, matching-to-sample discriminations.

\section{Apparatus}

The experiment was conducted in a BRS/LVE (Laurel, MD) sound-attenuating pigeon test chamber. The operant chamber measured $32 \mathrm{~cm}$ high, $32 \mathrm{~cm}$ across the response panel, and $28 \mathrm{~cm}$ from the response panel to the back wall. Three square response keys $(2.5 \times 2.5 \mathrm{~cm})$ were aligned horizontally on the response panel and were separated by $0.8 \mathrm{~cm}$. Only the left and right response keys were used in this experiment. The bottom edge of the response keys was $25.5 \mathrm{~cm}$ from the wire-mesh floor. A 12-stimulus in-line projector (Industrial Electronics Engineering, Van Nuys, CA) with 28-V, 0.1-A lamps (GE 1820) was mounted behind each response key. The left and right response keys projected two hues, red and green (Kodak Wratten Filters 26 and 60, respectively).

The sample stimulus consisted of an unfiltered houselight (GE 1820) mounted in the center of the chamber ceiling. A second, shielded houselight (GE 1820) was located above the center response key, $3.5 \mathrm{~cm}$ from the ceiling of the chamber. It was surrounded by a blue filter (Kodak Wratten Filter 38A). A rear-mounted feeder provided mixed grain reinforcement (Purina Pro Grains) through a $5.1 \times 5.5 \mathrm{~cm}$ aperture centered horizontally on the response panel and vertically midway between the response keys and the floor of the chamber. Reinforcement consisted of 2-sec access to mixed grain. An exhaust fan mounted on the outside of the chamber masked extraneous noise. The experiment was controlled and data collected by a microcomputer located in an adjacent room.

\section{Procedure}

Training. Two groups of pigeons were placed immediately into training on duration sample matching. For Group ITI-Dark $(n=4)$, the ITI consisted of a 15-sec period of darkness. For Group ITI-Lit $(n=4)$, the second, filtered houselight was illuminated during the 15-sec ITI. Each trial began with illumination of the unfiltered houselight, which served as the sample, for 0,2 , or $10 \mathrm{sec}$. Offset of the sample was followed by a 1-sec dark retention interval. This period of darkness was 

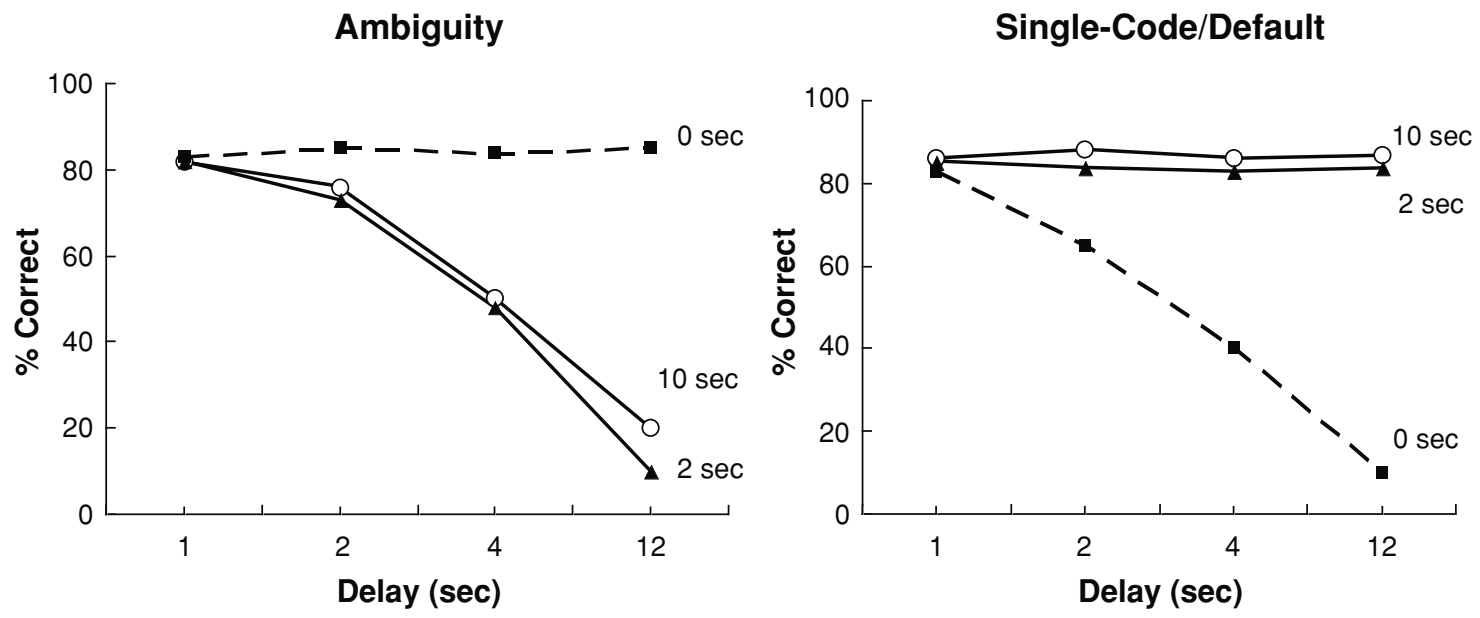

\begin{abstract}
Figure 1. Predicted results following hybrid one-to-one/many-to-one matching with 0-sec samples (squares) associated with one comparison and 2- and 10-sec samples (circles and triangles, respectively) associated with the other comparison. Retention functions predicted by the ambiguity hypothesis with dark intertrial intervals and dark retention intervals are presented on the left. Those predicted by a single-code/default hypothesis with lit intertrial intervals and dark retention intervals are presented on the right.
\end{abstract}

followed by presentation of red and green comparison stimuli on the side response keys. For half of the pigeons in each group, choice of the red comparison was reinforced following a 0 -sec sample, and choice of the green comparison was reinforced following both a 2 - and a 10 -sec sample. For the remaining pigeons, the contingencies were reversed. A 15-sec ITI followed all comparison choices. Each training session consisted of 64 trials, with thirty-two 0 -sec sample trials, sixteen 2-sec sample trials, and sixteen 10 -sec sample trials per session. Thus, there were equal numbers of red and green correct side key trials in each session. Training sessions were conducted once a day, 6 days a week.

During training, a correction procedure was in effect. If a pigeon chose the incorrect comparison, after a 15 -sec ITI, the trial was repeated to a maximum of five times. On a repeated trial, a correct response was not reinforced, but the feeder light was illuminated. Performance on correction trials was not included in session performance scores.

The pigeons were trained to a criterion of $80 \%$ correct or better on each of the three sample types for five consecutive sessions. When criterion was reached, the correction procedure was discontinued, and training continued until the birds reached a criterion of $75 \%$ or better on all three samples for two consecutive sessions.

Testing. Testing sessions were similar to training sessions, with the exception that the duration of the retention interval was $1,2,4$, or 12 sec. There were 16 trials at each retention interval, 8 involving 0 -sec samples, 4 involving 2-sec samples, and 4 involving 10 -sec samples, randomly presented in each testing session. A total of 10 testing sessions were conducted, with 2 days of training following each testing session.

\section{Results}

\section{Acquisition}

The pigeons reached criterion in an average of 33 sessions $(M=33.13, S E M=3.04)$. There was no significant difference in rate of acquisition for the pigeons in Group ITI-Dark and Group ITI-Lit $(F=1.82, p>.05)$.

\section{Retention Testing for Group ITI-Dark}

Matching accuracy during testing for Group ITI-Dark on 0 -sec sample trials remained consistently high with increasing retention interval, whereas matching accuracy on 2 - and 10-sec sample trials declined rapidly with increas- ing retention interval, to a level below chance. The test session data from Group ITI-Dark are presented in the left panel of Figure 2.

We performed a two-way repeated measures ANOVA on the testing data from Group ITI-Dark, with sample duration $(0,2$, and $10 \mathrm{sec})$ and retention interval $(1,2,4$, and $12 \mathrm{sec})$ as factors. There was a significant effect of sample duration $[F(2,6)=19.30, p<.01]$ and a significant effect of retention interval $[F(3,9)=116.62, p<.01]$. There was also a significant sample duration $\times$ retention interval interaction $[F(6,18)=19.16, p<.01]$. As can be seen in the left panel of Figure 2, the interaction can be attributed to the divergent retention functions for the 0 -sec sample trials versus those for the 2 - and 10 -sec sample trials.

\section{Retention Testing for Group ITI-Lit}

Matching accuracy during testing for Group ITI-Lit on 0 -sec sample trials was high, whereas performance on 2- and 10-sec sample trials dropped below chance. A two-way repeated measures ANOVA was performed on the pooled test data. As with Group ITI-Dark, there was a significant effect of sample duration $[F(2,6)=22.97, p<$ $.01]$ and a significant effect of retention interval $[F(3,9)=$ $32.04, p<.01]$. A significant sample duration $\times$ retention interval interaction was also found $[F(6,18)=12.70, p<$ $.01]$. As is shown in the right panel of Figure 2, this interaction can be attributed to the divergent retention functions for 0 -sec sample trials versus those for 2 - and 10 -sec sample trials.

\section{Discussion}

Although the saliency of the sample was increased from that in Zentall et al. (2004) by replacing the blue houselight with the white houselight, the retention functions from neither group support the hypothesis that pigeons used a single-code/default strategy in this three-sample- 

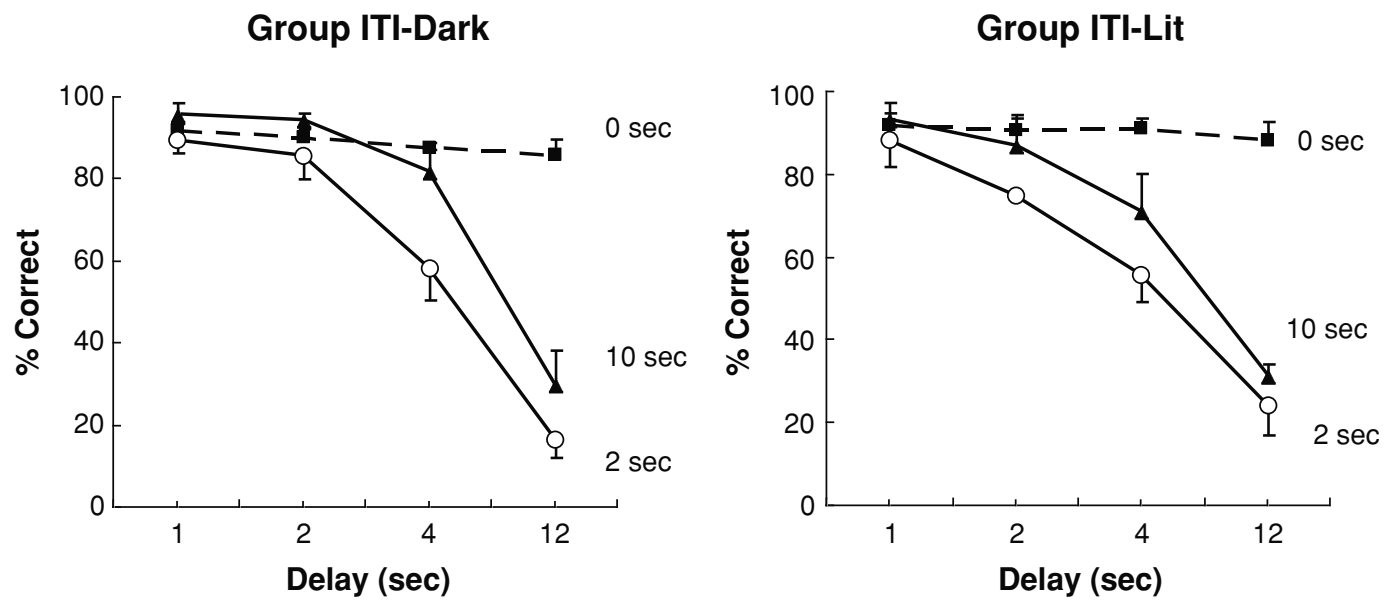

Figure 2. Experiment 1: Retention function (means with $S E M$ s) following hybrid one-to-one/many-to-one matching with 0 -sec samples (squares) associated with one comparison and 2 - and 10-sec samples (circles and triangles, respectively) associated with the other comparison. Data from the group that had the dark intertrial interval (Group ITI-Dark) are presented on the left. Data from the group that had the lit ITI (Group ITI-Lit) are presented on the right.

duration matching task. If a single-code/default strategy had been used, matching accuracy on 0 -sec sample trials should have decreased with increasing retention interval, whereas matching accuracy on 2- and 10-sec sample trials should have remained high. However, the opposite result was obtained.

As was predicted, retention functions for Group ITIDark were consistent with the ambiguity hypothesis. The pigeons responded as though all the trials at long delays were 0 -sec sample trials, suggesting that long delays were treated as 0 -sec sample trials. However, similar retention functions were also found for Group ITI-Lit. The use of a houselight to illuminate the ITI should have prevented ambiguity between the ITI and the retention interval. Instead, it appears that the use of a 0 -sec sample may have created a task similar to present/absent sample matching (Sherburne \& Zentall, 1993). Pigeons could have used the rule, "If a sample light comes on, choose red; if no sample light comes on, choose green." This explanation is consistent with the detection hypothesis presented by Wixted and Gaitan (2004), in which the most salient sample (presence of a houselight) was the coded sample. This may be a more efficient strategy than using a single-code/default strategy, in which the single sample associated with one comparison was detected and remembered, whereas the comparison associated with the other two samples was chosen by default. Thus, the pigeons could have coded the 2 - and 10-sec samples together as sample present and the 0 -sec sample as sample absent. It may be that the use of a 0 -sec sample produces a unique absent-sample condition in which a more efficient strategy (i.e., present/absent matching) was used.

One way to correct this problem would be to use three nonzero duration samples, two associated with one comparison and a third associated with the other. With three nonzero sample durations, the pigeons should be less likely to use present/absent as the basis for the discrimination. In this case, the most efficient strategy might be to code the sample associated with the single comparison and to respond by default on trials involving the remaining two samples.

\section{EXPERIMENT 2}

In Experiment 2, three nonzero durations were used (2, 8 , and $32 \mathrm{sec}$ ). If, under these conditions, pigeons use a single-code/default strategy, testing with retention intervals should result in divergent retention functions, with matching accuracy on trials involving the two sample durations associated with the same comparison remaining high and flat and matching accuracy on trials involving the third sample declining with increasing retention intervals. According to the ambiguity hypothesis, if divergent retention functions were found for pigeons in Group ITIDark, it would still be possible that the dark ITI and dark retention intervals were confused. However, in this case, one would have to assume that the presumed absence of a sample on delay trials was sufficiently similar to a 2 -sec sample from training to result in choice of the comparison associated with that sample. If ambiguity between the ITI and the retention interval were the only biasing factor present, however, one would expect to find parallel retention functions for Group ITI-Lit, because for this group, the easily discriminated lit ITI and the dark retention interval should not be confused. Thus, divergent retention functions for Group ITI-Lit would suggest the development of a single-code/default coding strategy.

In Experiment 2, we trained the pigeons to choose one comparison stimulus following presentation of the shortest (2-sec) sample and the longest (32-sec) sample and to choose the other comparison stimulus following presentation of the middle duration $(8-\mathrm{sec})$ sample. We used these 
pairings in training to preclude the association of the two shortest samples or the two longest samples with the same comparison stimulus.

\section{Method}

Subjects and Apparatus

The 8 pigeons used in Experiment 2 were similar to those used in Experiment 1. The apparatus was the same as that used in Experiment 1.

\section{Procedure}

The procedure used in Experiment 2 for Group ITI-Dark and Group ITI-Lit was similar to that used in Experiment 1, with the following differences. In Experiment 2, the samples consisted of illumination of the white houselight for 2,8 , or $32 \mathrm{sec}$. For half of the pigeons in each group, choice of the red comparison was reinforced following an 8-sec sample, and choice of the green comparison was reinforced following both a 2 - and a 32 -sec sample. For the remaining pigeons, the samples associated with the red and green comparisons were reversed. Testing sessions were similar to those conducted in Experiment 1. However, in Experiment 2, matching accuracy was assessed at five different retention intervals $(1,2,6,14$, and $30 \mathrm{sec})$. There were 80 trials in each testing session, with 16 trials at each retention interval.

\section{Results \\ Acquisition \\ The pigeons reached the training criterion in an average of 56 sessions $(M=55.75, S E M=7.16)$. There was no significant difference in acquisition rates for the pigeons in Group ITI-Dark and Group ITI-Lit $(F=2.02, p>.05)$. It did, however, take the pigeons in Experiment 2 signifi- cantly longer to learn the task than it took the pigeons in Experiment $1(F=8.47, p=.01)$.}

\section{Retention Testing for Group ITI-Dark}

During testing, matching accuracy for the pigeons in Group ITI-Dark on both 2- and 32-sec sample trials remained consistently high with an increasing retention in- terval, whereas matching accuracy on 8-sec sample trials declined rapidly, to a level below chance. The test session data from Group ITI-Dark are presented in the left panel of Figure 3.

We performed a two-way repeated measures ANOVA on the testing data from Group ITI-Dark, with sample duration $(2,8$, and $32 \mathrm{sec})$ and retention interval $(1,2$, $6,14$, and $30 \mathrm{sec})$ as factors. There was a significant effect of sample duration $[F(2,6)=11.58, p<.01]$ and a significant effect of retention interval $[F(4,12)=16.40$, $p<.01]$. There was also a significant sample duration $\times$ retention interval interaction $[F(8,24)=10.06, p<.01]$. As is shown in the left panel of Figure 3, the interaction can be attributed to the divergent retention functions for the 8-sec sample trials versus those for the 2- and 32-sec sample trials.

\section{Retention Testing for Group ITI-Lit}

During testing, matching accuracy for the pigeons in Group ITI-Lit on both 2- and 32-sec samples remained high, whereas performance on 8-sec sample trials dropped below chance (see the right panel of Figure 3). A two-way repeated measures ANOVA was performed on the testing data. As for Group ITI-Dark, there was a significant effect of retention interval $[F(4,12)=34.33, p<.01]$. Although the main effect of sample duration was not statistically significant $[F(2,6)=3.74, p=.088]$, there was a significant sample duration $\times$ retention interval interaction $[F(8,24)=2.45, p<.05]$. Again, the interaction can be attributed to the divergent retention functions for the 8-sec sample trials versus those for the 2- and 32-sec sample trials.

\section{Discussion}

The retention functions for both groups revealed a similar pattern of results. Matching accuracy on the 8-sec sample trials decreased with an increasing retention in-
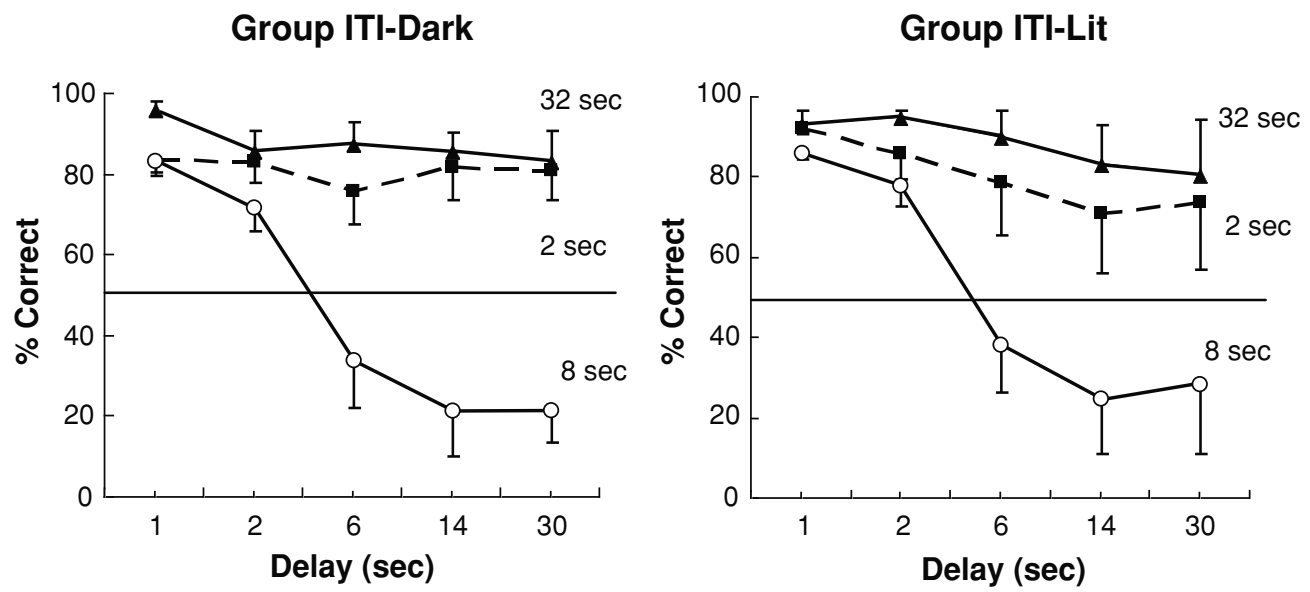

Figure 3. Experiment 2: Retention function (means with $S E M$ s) following hybrid one-to-one/manyto-one matching with 8-sec samples (circles) associated with one comparison and 2- and 32-sec samples (squares and triangles, respectively) associated with the other comparison. Data from the group that had the dark intertrial interval (Group ITI-Dark) are presented on the left. Data from the group that had the lit ITI (Group ITI-Lit) are presented on the right. 
terval, whereas matching accuracy on the 2- and 32-sec sample trials remained high. Because of the potential ambiguity between the ITI and the retention interval, it is difficult to interpret the divergent retention functions found for Group ITI-Dark. However, for Group ITI-Lit, lighting the ITI should have made it distinct from the dark retention interval, thereby removing the ambiguity. For this reason, these results suggest that in the absence of retention interval ambiguity, the task demands encourage the development of an efficient single-code/default coding strategy. Thus, for the first time, we have found evidence for the use of a single-code/default coding strategy in duration sample discriminations in the absence of either ITIretention-interval confusion or a present/absent discrimination produced by using a 0 -sec sample. It is possible that the pigeons in Group ITI-Dark were also using a singlecode/default strategy; however, it is unclear whether the divergent functions were a result of this coding strategy or an artifact of retention interval ambiguity.

\section{GENERAL DISCUSSION}

Gaitan and Wixted (2000) proposed that pigeons could use a detection strategy to acquire a conditional discrimination involving three duration samples, two associated with one comparison, one associated with the other. With such a strategy, pigeons learn to detect one sample and to respond by default in its absence. Such a strategy may develop because it is more efficient than learning three different sample-comparison associations. Zentall et al. (2004) offered an alternative account for the divergent retention functions found by Gaitan and Wixted and proposed that the results could be an artifact of the ambiguity between dark ITIs and retention intervals. Zentall et al. found that under similar conditions, disambiguating the ITI and retention interval by lighting the ITI resulted in parallel, rather than divergent, retention functions.

In Experiment 1 of the present study, we attempted to find evidence for the development of a single-code/ default (or detection) coding strategy by making the sample durations more salient. Under these conditions, matching accuracy on 0 -sec sample trials remained accurate over retention intervals, but performance on both 2- and 10-sec sample trials declined substantially. Because the resultant divergent retention functions were similar to those typically found in present/absent matching, we concluded that the use of a 0 -sec sample was inappropriate for the study of duration sample retention.

In Experiment 2, three nonzero duration samples were used. This allowed us to reduce the likelihood that the pigeons would treat the task as presence/absence sample matching. Furthermore, the middle duration sample was the one uniquely associated with one comparison, whereas both the shorter and the longer samples were associated with the other comparison. In this way, the pigeons could not group those two samples solely by their durations, relative to the middle duration sample. Although the pigeons in Group ITI-Dark may have confused the novel retention intervals with the familiar ITIs, that should not have been true for Group ITI-Lit.

Thus, the results of Experiment 2 are consistent with the development of a single-code/default coding strategy. The pigeons appeared to remember the 8-sec sample that was associated with one comparison and to choose the other comparison by default when there was no memory of an 8-sec sample. For this reason, over long retention intervals, accuracy on both 2 - and 32-sec sample trials remained high and flat, and accuracy on 8-sec sample trials decreased to a level below chance. The present results suggest that pigeons have considerable flexibility in the development of coding strategies, and they support earlier evidence that under appropriate conditions, pigeons can develop a single-code/default coding strategy (Clement \& Zentall, 2000) in the absence of event ambiguity or the use of an absent event.

It should be noted that although the results of Experiment 2 are consistent with the development of a singlecode/default response, it is not likely that such a mechanism can account for the divergent retention functions typically found in duration sample discriminations in which only two samples were used. For example, Grant and Spetch (1994) trained pigeons on a duration sample discrimination involving 2 - versus 10 -sec samples with color comparisons and another duration sample discrimination involving 4.5- versus 22.5 -sec samples with line orientation comparisons. When the pigeons were tested with the two comparisons associated in training with the short samples, they showed better-than-chance choice of the comparison that in training was associated with the appropriate comparison (i.e., the color following the 2 -sec sample and the line orientation following the 4.5-sec sample). If the pigeons had acquired these discriminations using a single-code/default response, they should have chosen the two comparisons equally, because both would have been appropriate for the short default sample. Similar results were found when the pigeons were tested with the two comparisons associated in training with the long samples (i.e., the color following the 10-sec sample and the line orientation following the 22.5-sec sample). Thus, the correct comparisons on long sample trials as well were not chosen by default.

The results from Grant and Spetch (1994), together with the effects of lighting the ITI when the delay is dark, suggest that duration sample discriminations are not typically acquired by representing one of the samples and choosing the alternative comparison by default; however, the present results suggest that under certain conditions, particularly when more than one sample is associated with the same comparison, the development of a single-code/ default response may be encouraged.

\section{REFERENCES}

Clement, T. S., \& Zentall, T. R. (2000). Development of a singlecode/default coding strategy in pigeons. Psychological Science, 11, 261-264.

Colwill, R. M. (1984). Disruption of short-term memory for reinforce- 
ment by ambient illumination. Quarterly Journal of Experimental Psychology, 36B, 235-258.

GaITAN, S. C., \& WiXTED, J. T. (2000). The role of "nothing" in memory for event duration in pigeons. Animal Learning \& Behavior, 28, 147161.

GRANT, D. S. (1991). Symmetrical and asymmetrical coding of food and no-food samples in delayed matching in pigeons. Journal of Experimental Psychology: Animal Behavior Processes, 17, 243-259.

GrAnt, D. S., \& SPETCH, M. L. (1993). Analogical and nonanalogical coding of samples differing in duration in a choice-matching task in pigeons. Journal of Experimental Psychology: Animal Behavior Processes, 19, 15-25.

Grant, D. S., \& SPETCH, M. L. (1994). The role of asymmetrical coding of duration samples in producing the choose-short effect in pigeons. Learning \& Motivation, 25, 413-430.

KelLy, R., \& SPETCH, M. L. (2000). Choice biases in delayed matchingto-sample duration with pigeons: Manipulation of ITI and delay illumination. Quarterly Journal of Experimental Psychology, 53B, 309-323.

Sherburne, L. M., \& Zentall, T. R. (1993). Coding of feature and no-feature events by pigeons performing a delayed conditional discrimination. Animal Learning \& Behavior, 21, 92-100.

Sherburne, L. M., Zentall, T. R., \& Kaiser, D. H. (1998). Timing in pigeons: The choose-short effect may result from pigeons' "confu- sion" between delay and intertrial intervals. Psychonomic Bulletin \& Review, 5, 516-522.

SPETCH, M. L. (1987). Systematic errors in pigeons' memory for event duration: Interaction between training and test delay. Animal Learning \& Behavior, 15, 1-5.

SPETCH, M. L., \& WiLKIE, D. M. (1983). Subjective shortening: A model of pigeons' memory for event duration. Journal of Experimental Psychology: Animal Behavior Processes, 9, 14-30.

Wilson, B., \& BOAKES, R. A. (1985). A comparison of the short-term memory performances of pigeons and jackdaws. Animal Learning \& Behavior, 13, 285-290.

Wixted, J. T., \& Gaitan, S. C. (2004). Stimulus salience and asymmetric forgetting in the pigeon. Learning \& Behavior, 32, 173-182.

Zentall, T. R., Klein, E. D., \& Singer, R. A. (2004). Evidence for detection of one duration sample and default responding to other duration samples by pigeons may result from an artifact of retention-test ambiguity. Journal of Experimental Psychology: Animal Behavior Processes, 30, 129-134.

(Manuscript received May 3, 2006; revision accepted for publication July 10, 2006.) 